MATHEMATICS OF COMPUTATION

Volume 73, Number 248, Pages 1903-1911

S 0025-5718(04)01653-9

Article electronically published on April 22, 2004

\title{
ON STRONG TRACTABILITY OF WEIGHTED MULTIVARIATE INTEGRATION
}

\author{
FRED J. HICKERNELL, IAN H. SLOAN, AND GRZEGORZ W. WASILKOWSKI
}

\begin{abstract}
We prove that for every dimension $s$ and every number $n$ of points, there exists a point-set $\mathcal{P}_{n, s}$ whose $\boldsymbol{\gamma}$-weighted unanchored $L_{\infty}$ discrepancy is bounded from above by $C(b) / n^{1 / 2-b}$ independently of $s$ provided that the sequence $\boldsymbol{\gamma}=\left\{\gamma_{k}\right\}$ has $\sum_{k=1}^{\infty} \gamma_{k}^{a}<\infty$ for some (even arbitrarily large) $a$. Here $b$ is a positive number that could be chosen arbitrarily close to zero and $C(b)$ depends on $b$ but not on $s$ or $n$. This result yields strong tractability of the corresponding integration problems including approximation of weighted integrals $\int_{D} f(\mathbf{x}) \rho(\mathbf{x}) d \mathbf{x}$ over unbounded domains such as $D=\mathbb{R}^{s}$. It also supplements the results that provide an upper bound of the form $C \sqrt{s / n}$ when $\gamma_{k} \equiv 1$.
\end{abstract}

\section{INTRODUCTION}

This article studies the unanchored $L_{\infty}$ discrepancy and strong tractability of the corresponding integration problem. We begin the discussion with the integration problem. Consider approximating the following type of weighted integrals:

$$
I_{\rho}(f)=\int_{D} f(\mathbf{x}) \rho(\mathbf{x}) d \mathbf{x} .
$$

Here $D$ is an $s$-dimensional box,

$$
D=\overline{\left(a_{1}, b_{1}\right)} \times \cdots \times \overline{\left(a_{s}, b_{s}\right)} \subseteq \mathbb{R}^{s},
$$

with possibly infinite $a_{i}$ and/or $b_{i}$. It is assumed that the weight function $\rho$ has a tensor product form,

$$
\rho(\mathbf{x})=\prod_{k=1}^{s} \rho_{k}\left(x_{k}\right)
$$

for nonnegative and Lebesgue integrable functions $\rho_{k}$. For simplicity, it is assumed that the $\rho_{k}$ are probability densities on $\overline{\left(a_{k}, b_{k}\right)}$, i.e.,

$$
\int_{a_{k}}^{b_{k}} \rho_{k}(x) d x=1 .
$$

However, as explained in Section 6.1 in [3], it is sufficient to assume that the integrals of $\rho_{k}$ are finite.

Received by the editor December 16, 2002 and, in revised form, April 30, 2003.

2000 Mathematics Subject Classification. Primary 65D30, 65D32, 65Y20, 11 K38.

Key words and phrases. Weighted integration, quasi-Monte Carlo methods, low discrepancy points, tractability.

(C)2004 American Mathematical Society 
Without loss of generality, the analysis can be restricted to linear algorithms

$$
\mathcal{A}_{\mathcal{P}_{n, s}}(f)=\sum_{i=1}^{n} w_{i} f\left(\mathbf{x}^{i}\right)
$$

where $\mathcal{P}_{n, s}=\left\{\mathbf{x}^{1}, \ldots, \mathbf{x}^{n}\right\}$ is a set of points from $D$ and the $w_{i}$ are numbers. Important examples of such algorithms are provided by the quasi-Monte Carlo methods, denoted by $\mathcal{Q}_{\mathcal{P}_{n, s}}$,

$$
\mathcal{Q}_{\mathcal{P}_{n, s}}(f)=n^{-1} \sum_{i=1}^{n} f\left(\mathbf{x}^{i}\right)
$$

For a given normed space $\mathcal{F}$ of integrands, the error of $\mathcal{A}_{\mathcal{P}_{n, s}}$ is given by

$$
\operatorname{error}\left(\mathcal{A}_{\mathcal{P}_{n, s}} ; \mathcal{F}\right):=\sup _{\|f\| \leq 1}\left|I_{\rho}(f)-\mathcal{A}_{\mathcal{P}_{n, s}}(f)\right|=\left\|I_{\rho}-\mathcal{A}_{\mathcal{P}_{n, s}}\right\|,
$$

where $\|f\|$ is the norm of $f$ in the space $\mathcal{F}$. The importance of this definition is that

$$
\left|I_{\rho}(f)-\mathcal{A}_{\mathcal{P}_{n, s}}(f)\right| \leq\|f\| \operatorname{error}\left(\mathcal{A}_{\mathcal{P}_{n, s}} ; \mathcal{F}\right), \quad \forall f \in \mathcal{F} .
$$

Let $\operatorname{error}(n ; \mathcal{F})$ denote the $n$th minimal error, i.e., the minimal error among all algorithms that use at most $n$ function evaluations,

$$
\operatorname{error}(n ; \mathcal{F}):=\inf _{\mathcal{A}_{\mathcal{P}_{n}, s}} \operatorname{error}\left(\mathcal{A}_{\mathcal{P}_{n, s}} ; \mathcal{F}\right) \quad \text { and } \operatorname{error}(0 ; \mathcal{F}):=\left\|I_{\rho}\right\| \text {. }
$$

We say that the corresponding problem is tractable if there exist nonnegative constants $C, q_{1}$, and positive $q_{2}$ such that

$$
\frac{\operatorname{error}(n ; \mathcal{F})}{\operatorname{error}(0 ; \mathcal{F})} \leq C s^{q_{1}} n^{-q_{2}}, \quad \forall n, s \geq 1 .
$$

Moreover, the problem is strongly tractable if (4) holds with $q_{1}=0$.

In an earlier paper [3], we considered this problem in the space $\mathcal{F}=\mathcal{F}_{1,1, s}$ (see the next section for a formal definition), whose norm is given by

$$
\|f\|_{1,1, s}:=|f(\mathbf{c})|+\sum_{U \neq \emptyset}\left\|f_{U}^{\prime}\right\|_{L_{1}\left(D_{U}\right)} .
$$

Here the point $\mathbf{c}$, called the anchor, is a fixed point from $D$. The summation is over nonempty subsets $U$ of $\{1, \ldots, s\}$, and $f_{U}^{\prime}$ is obtained from $\left(\prod_{k \in U} \frac{\partial}{\partial x_{k}}\right) f$ by fixing the variables $x_{k}$ for $k \notin U$ and replacing them by the $k$ th coefficients of the anchor $\mathbf{c}$. Moreover, $D_{U}$ is the Cartesian product $\prod_{k \in U} \overline{\left(a_{k}, b_{k}\right)}$ and $\mathbf{x}_{U}$ is the projection of $\mathbf{x}$ onto $D_{U}$. Note that for $D=[0,1]^{s}$ and $\mathbf{c}=\mathbf{1}$, this is a classical norm often assumed in the theory of low discrepancy points and multidimensional integration. Using a proof technique similar to the one used in [2], we proved tractability of weighted integration for any $\rho$ satisfying the above assumptions. That is, in particular, we showed that there exists a constant $C$ such that

$$
\operatorname{error}\left(n ; \mathcal{F}_{1,1, s}\right) \leq C \sqrt{\frac{s}{n}}, \quad \forall n, s \geq 1 .
$$

Since the initial error $\operatorname{error}\left(0 ; \mathcal{F}_{1,1, s}\right)$ equals 1 , this implies tractability of the weighted integration problem for the space $\mathcal{F}_{1,1, s}$.

Although we doubt that the upper bound (66) is sharp, it was also shown in [2] that the dependence on $s$ cannot be eliminated. It is therefore natural to ask under what restrictions on the class $\mathcal{F}_{1,1, s}$ the corresponding $n$th minimal errors 
can be bounded from above independently of $s$, i.e., when the integration problem is strongly tractable.

The main result of this paper provides a partial answer to this question by considering so-called weighted spaces introduced in [9] and then further studied in a number of papers. More specifically, let $\gamma=\left\{\gamma_{k}\right\}_{k}$ be a sequence of positive numbers (called weights). For simplicity, we assume that $\gamma_{k} \leq 1$. Then the space $\mathcal{F}_{1, \gamma, s}$ is a Banach space whose norm is given by

$$
\|f\|_{1, \gamma, s}:=|f(\mathbf{c})|+\sum_{U \neq \emptyset} \gamma_{U}^{-1}\left\|f_{U}^{\prime}\right\|_{L_{1}\left(D_{U}\right)},
$$

where

$$
\gamma_{U}:=\prod_{k \in U} \gamma_{k} \quad \text { and } \quad \gamma_{\emptyset}:=1
$$

Note that for $\gamma_{k} \equiv 1$, the norms (5) and (7) coincide. Moreover, also for arbitrary $\gamma$ we have $\operatorname{error}\left(0 ; \mathcal{F}_{1, \gamma, s}\right)=1$.

We prove that the $n$th minimal errors are arbitrarily close to a constant times $n^{-1 / 2}$ (independently of $s$ ) provided that

$$
\sum_{k=1}^{\infty} \gamma_{k}^{a}<\infty
$$

for a positive (even arbitrarily large) number $a$. For instance, condition (8) is satisfied if $\gamma_{k}=O\left(1 / k^{\delta}\right)$ for a positive (even arbitrarily small) number $\delta$.

That is, we show that (8) implies the following: for every $b>0$ there exists a constant $C(b)$ such that

$$
\operatorname{error}\left(n ; \mathcal{F}_{1, \boldsymbol{\gamma}, s}\right) \leq C(b) n^{-1 / 2+b}, \quad \forall n, s \geq 1 .
$$

This is proved by showing an existence of quasi-Monte Carlo algorithms $\mathcal{Q}_{\mathcal{P}_{n, s}}$ with errors bounded from above by $C(b) n^{-1 / 2+b}$.

Actually, we show an even stronger result by considering the so-called unanchored $L_{\infty}$ discrepancy $\mathcal{D}_{\infty}\left(\mathcal{P}_{n, s}, \gamma\right)$. Its definition is presented in the next section; here we only mention that for every anchor $\mathbf{c}$, every sequence $\boldsymbol{\gamma}$, and every $\mathcal{P}_{n, s}$, we have

$$
\operatorname{error}\left(\mathcal{Q}_{\mathcal{P}_{n, s}} ; \mathcal{F}_{1, \gamma, s}\right) \leq \mathcal{D}_{\infty}\left(\mathcal{P}_{n, s}, \gamma\right) .
$$

Our main result is as follows.

Main Theorem. Let (8) hold for some $a>0$. Then for any positive $b$ there exists a constant $C(b)$ such that for every $n$ and $s$ there is $\mathcal{P}_{n, s}$ with

$$
\mathcal{D}_{\infty}\left(\mathcal{P}_{n, s}, \gamma\right) \leq C(b) n^{b-1 / 2} .
$$

This and (10) imply that the weighted integration problem for the space $\mathcal{F}_{1, \boldsymbol{\gamma}, s}$ is strongly tractable under a very mild assumption concerning the convergence of the $\gamma_{k}$ 's. This is in contrast to tractability and strong tractability results obtained for weighted classes with $L_{1}$-norm replaced by $L_{p}$-norm $(p>1)$. Indeed, let $\mathcal{F}_{p, \gamma, s}$ be the space equipped with the norm

$$
\|f\|_{p, \boldsymbol{\gamma}, s}:=\left(\sum_{U} \gamma_{U}^{-p}\left\|f_{U}^{\prime}\right\|_{L_{p}}^{p}\right)^{1 / p} \quad \text { for } p<\infty
$$

and

$$
\|f\|_{\infty, \boldsymbol{\gamma}, s}:=\max _{U} \gamma_{U}^{-1}\left\|f_{U}^{\prime}\right\|_{L_{\infty}}
$$


Then for $p>1$, a necessary and sufficient condition for tractability of the integration problem for the space $\mathcal{F}=\mathcal{F}_{p, \boldsymbol{\gamma}, s}$ (with an arbitrary anchor) is that

$$
\sup _{s \geq 2} \frac{\sum_{k=1}^{s} \gamma_{k}^{p^{*}}}{\ln (s)}<\infty
$$

where $p^{*}$ is the conjugate to $p, 1 / p^{*}=1-1 / p$. For strong tractability, it is necessary and sufficient that

$$
\sum_{k=1}^{\infty} \gamma_{k}^{p^{*}}<\infty
$$

see [4, 7]. The results for $p>1$ suggest that condition (8) might be necessary for strong tractability as well.

For every point-set $\mathcal{P}_{n, s}$, its unanchored discrepancy is an upper bound on its same-quadrant and star discrepancies. Hence the upper bound (11) holds for these discrepancies as well. The star discrepancy (with $\gamma_{k} \equiv 1$ ), denoted by $\mathcal{D}_{\infty}^{*}\left(\mathcal{P}_{n, s}\right)$, has been analyzed in many papers, and many deep and important results are available. In particular, it is known (see, e.g., [1, 5]) that there are point-sets $\mathcal{P}_{n, s}$ for which

$$
\mathcal{D}_{\infty}^{*}\left(\mathcal{P}_{n, s}\right) \leq c(s) \frac{(\ln n)^{s-1}}{n}
$$

where $c(s)$ depends on $s$ but not on $n$. Such bounds are sharper than (6) and (11) when $n$ is very large relative to $s$. Note, however, that $(\ln n)^{s-1} / n$ is an increasing function of $n$ when $n \leq \exp (s-1)$ and that $\exp (s-1)$ could be very large even for relatively small $s$. Hence, for problems with not too small $s$ and/or not too large $n$, the bounds (6) and (11) are sharper.

We now summarize the content of this paper. In Section 2, we provide basic definitions and facts. The proof of the main theorem is presented in Section 3.

\section{BASIC DEFINITIONS AND FACTS}

In this section we briefly recall basic facts and definitions used in this paper. For more detailed discussion on discrepancies and the worst case setting, we refer the reader to [1, 5] and to [6] 10, respectively.

Recall that we are interested in approximating integrals $I_{\rho}$ given by (1)-(3) in the worst case setting with respect to the Banach space $\mathcal{F}=\mathcal{F}_{1, \gamma, s}$ whose formal definition is presented now.

Let $\mathcal{H}^{s}$ be the linear space generated by the linear combinations of functions

$$
f(\mathbf{x})=\prod_{k=1}^{s} h_{k}\left(x_{k}\right)
$$

where for every $k, h_{k}: \overline{\left(a_{k}, b_{k}\right)} \rightarrow \mathbb{R}$ is an absolutely continuous function with $h_{k}^{\prime} \in L_{1}\left(\overline{\left(a_{k}, b_{k}\right)}\right)$. Then, for a given sequence $\gamma$, the number $s$ of variables, and the anchor $\mathbf{c}$, the space $\mathcal{F}_{1, \gamma, s}$ is the completion of $\mathcal{H}^{s}$ with respect to the norm $\|\cdot\|_{1, \gamma, s}$ given by (7).

As shown in [3], the weighted integration problem $I_{\rho}$ for the space $\mathcal{F}_{1, \boldsymbol{\gamma}, s}$ has the same complexity as the complexity of unweighted integration on the unit cube for the space $\mathcal{F}$ defined as $\mathcal{F}_{1, \gamma, s}$, however, with $D$ replaced by the unit cube and (in general) a different anchor. Moreover, for a quasi-Monte Carlo method for one problem, there exists a quasi-Monte Carlo method for the other problem, both with identical worst case errors. (Formally, this has been shown in [3] only for $\gamma_{k} \equiv 1$; 
however, the proof extends trivially to any $\gamma$.) Hence, from now on, we can assume that

$$
\rho \equiv 1, \quad D=[0,1]^{s}, \quad \text { and } \quad \mathcal{P}_{n, s} \subset[0,1]^{s} .
$$

As follows from [3], the worst case error of $\mathcal{Q}_{\mathcal{P}_{n, s}}$ in the space $\mathcal{F}_{1, \gamma, s}$ is equal to the so-called (weighted) $L_{\infty}$ same-quadrant discrepancy of the point-set $\mathcal{P}_{n, s}=$ $\left\{\mathbf{x}^{1}, \ldots, \mathbf{x}^{n}\right\}$. That is,

$$
\operatorname{error}\left(\mathcal{Q}_{\mathcal{P}_{n, s}} ; \mathcal{F}_{1, \boldsymbol{\gamma}, s}\right)=\mathcal{D}_{\infty, \mathbf{c}}\left(\mathcal{P}_{n, s}, \gamma\right)
$$

where

$$
\mathcal{D}_{\infty, \mathbf{c}}\left(\mathcal{P}_{n, s}, \gamma\right):=\max _{U \neq \emptyset} \gamma_{U} \sup _{\mathbf{h} \in[0,1]^{s}}\left|\operatorname{disc}\left(B(\mathbf{h}, \mathbf{c}), U, \mathcal{P}_{n, s}\right)\right|
$$

with

$$
\operatorname{disc}\left(B(\mathbf{h}, \mathbf{c}), U, \mathcal{P}_{n, s}\right):=\operatorname{Vol}\left(B_{U}\left(\mathbf{h}_{U}, \mathbf{c}_{U}\right)\right)-n^{-1} \sum_{i=1}^{n} \mathbf{1}_{B_{U}\left(\mathbf{h}_{U}, \mathbf{c}_{U}\right)}\left(\mathbf{x}_{U}^{i}\right) .
$$

Here $B(\mathbf{h}, \mathbf{c})$ is the box with one corner at $\mathbf{h}$ and the opposite corner given by the unique vertex of $[0,1]^{s}$ that lies in the same-quadrant as $\mathbf{h}$ with respect to the anchor c. Of course, by $B_{U}\left(\mathbf{h}_{U}, \mathbf{c}_{U}\right)$ we mean the projection of $B(\mathbf{h}, \mathbf{c})$ onto $D_{U}$. See [3] for the precise definition.

Recall that the classical $L_{\infty}$-star discrepancy of $\mathcal{P}_{n, s}$ is given by

$$
\begin{aligned}
\mathcal{D}_{\infty}^{*}\left(\mathcal{P}_{n, s}\right) & :=\max _{U \neq \emptyset} \sup _{\mathbf{h} \in D}\left|\operatorname{disc}\left([\mathbf{0}, \mathbf{h}), U, \mathcal{P}_{n, s}\right)\right| \\
& =\sup _{\mathbf{h} \in D}\left|\operatorname{Vol}([\mathbf{0}, \mathbf{h}))-n^{-1} \sum_{i=1}^{n} \mathbf{1}_{[\mathbf{0}, \mathbf{h})}\left(\mathbf{x}^{i}\right)\right|,
\end{aligned}
$$

and its $\gamma$-weighted version is given by

$$
\mathcal{D}_{\infty}^{*}\left(\mathcal{P}_{n, s}, \gamma\right):=\max _{U \neq \emptyset} \gamma_{U} \sup _{\mathbf{h} \in D}\left|\operatorname{disc}\left([\mathbf{0}, \mathbf{h}), U, \mathcal{P}_{n, s}\right)\right| .
$$

By $[\mathbf{0}, \mathbf{h})$ we mean a box with opposite corners $\mathbf{0}$ and $\mathbf{h}$.

Of course, for every $\mathcal{P}_{n, s}$ and $\gamma$,

$$
\mathcal{D}_{\infty}^{*}\left(\mathcal{P}_{n, s}, \gamma\right)=\mathcal{D}_{\infty, 1}\left(\mathcal{P}_{n, s}, \gamma\right)
$$

i.e., the star discrepancy and the same-quadrant discrepancy coincide when $\mathbf{c}=\mathbf{1}$.

The (weighted) $L_{\infty}$ unanchored discrepancy is defined by

$$
\mathcal{D}_{\infty}\left(\mathcal{P}_{n, s}, \gamma\right):=\max _{U} \gamma_{U} \sup _{\mathbf{g}, \mathbf{h} \in[0,1]^{s}}\left|\operatorname{disc}\left([\mathbf{g}, \mathbf{h}), U, \mathcal{P}_{n, s}\right)\right|,
$$

where now $[\mathbf{g}, \mathbf{h})$ is the box with opposite corners $\mathbf{g}$ and $\mathbf{h}$.

It is easy to see that for every $\mathcal{P}_{n, s}$, every $\boldsymbol{\gamma}$, and every anchor $\mathbf{c}$,

$$
\mathcal{D}_{\infty, \mathbf{c}}\left(\mathcal{P}_{n, s}, \gamma\right) \leq \mathcal{D}_{\infty}\left(\mathcal{P}_{n, s}, \gamma\right), \quad \forall \mathbf{c} \in D
$$

which implies (10). 


\section{Proof of the Main Result}

We need the following three lemmas.

Lemma 1. The function $B(t):=t^{-2}((1+t) \ln (1+t)-t)(t>0)$ is decreasing and $\sup _{t>0} B(t)=1 / 2$.

Proof. Note that $\lim _{t \downarrow 0} B(t)=1 / 2$ and $B^{\prime}(t)=g(t) / t^{3}$, where $g(t)=2 t-$ $(2+t) \ln (1+t)$. Since $g^{\prime}(t)=t /(1+t)-\ln (1+t)$ and $g^{\prime \prime}(t)=-t /(1+t)^{2}<0 \forall t>0$, it follows that $g^{\prime}(t) \leq g^{\prime}(0)=0 \forall t>0$, and so $g(t) \leq g(0)=0 \forall t>0$. The conclusion of this lemma follows.

Lemma 2. If $c_{a}:=\sum_{k=1}^{\infty} \gamma_{k}^{a}<\infty$, then there exists $\hat{\gamma}<\infty$ such that

$$
\gamma_{U} \leq\left(c_{a} /|U|\right)^{|U| / a} \leq \hat{\gamma}, \quad \forall U \neq \emptyset .
$$

Proof. The proof of this inequality follows from the inequality relating the geometric mean to the arithmetic mean:

$$
\gamma_{U}=\prod_{k \in U} \gamma_{k}=\left[\prod_{k \in U} \gamma_{k}^{a}\right]^{1 / a} \leq\left[\frac{1}{|U|} \sum_{k \in U} \gamma_{k}^{a}\right]^{|U| / a} \leq\left(\frac{c_{a}}{|U|}\right)^{|U| / a} .
$$

Taking the limit of $|U| \rightarrow \infty$ of the right-hand side gives zero, so $\gamma_{U}$ is bounded above by some $\hat{\gamma}$.

Lemma 3. Under the conditions of Lemma 国 it follows that for $\delta<1$,

$$
\delta<\gamma_{U} \Rightarrow|U|<c_{a} e-a \ln (\delta) .
$$

Proof. Under the assumption that $\delta<\gamma_{U}$ and by Lemma 2 it follows that

$$
|U| \ln \left(|U| / c_{a}\right) \leq-a \ln \left(\gamma_{U}\right)<-a \ln (\delta) .
$$

Either $|U|<c_{a} e$ or $|U| \geq c_{a} e$. The former case is contained in the conclusion of this lemma. In the latter case the inequality above implies that $|U|<-a \ln (\delta)$.

We are ready to prove the theorem. The proof uses Bennett's inequality (see, e.g., [8]) and is somewhat similar to the proof of [2, Theorem 1]. Let $\delta=(\hat{c} / n)^{b}$ for some $b<1 / 2$ and some constant $\hat{c}$ independent of $n$ and $s$ to be determined later. It will be shown that there exists a set $\mathcal{P}_{n, s}$ with

$$
\mathcal{D}_{\infty}\left(\mathcal{P}_{n, s}, \gamma\right)=\max _{U} \sup _{\mathbf{g}, \mathbf{h} \in[0,1]^{s}} \gamma_{U}\left|\operatorname{disc}\left([\mathbf{g}, \mathbf{h}), U, \mathcal{P}_{n, s}\right)\right| \leq 3 \delta .
$$

For any $U \subseteq\{1, \ldots, s\}$, let $\delta_{U}=\delta / \gamma_{U}$. Since the absolute value of the local discrepancy function is no larger than 1 , it follows automatically that

$$
\sup _{\mathbf{g}, \mathbf{h} \in[0,1]^{s}} \gamma_{U}\left|\operatorname{disc}\left([\mathbf{g}, \mathbf{h}), U, \mathcal{P}_{n, s}\right)\right| \leq \gamma_{U} \leq \delta \quad \text { for } \quad \delta_{U} \geq 1 .
$$

Therefore, to prove (12), it is only necessary to consider those $U$ with $\delta_{U}<1$. For $U$ satisfying this condition, define $m_{U}=\left\lceil|U| / \delta_{U}\right\rceil$. Let $\Gamma_{U}$ be the equidistant grid $\left\{1 / m_{U}, \ldots,\left(m_{U}-1\right) / m_{U}\right\}^{|U|}$ in $D_{U}$. Of course, the cardinality of this grid is $\left|\Gamma_{U}\right|=\left(m_{U}-1\right)^{|U|}$. As in [2] it can be shown that for any nonempty $U$ and any set $\mathcal{P}_{n, s}$,

$$
\begin{gathered}
\sup _{\mathbf{g}, \mathbf{h} \in[0,1]^{s}}\left|\operatorname{disc}\left([\mathbf{g}, \mathbf{h}), U, \mathcal{P}_{n, s}\right)\right| \leq \frac{2|U|}{m_{U}}+\sup _{\mathbf{g}, \mathbf{h} \in \Gamma_{U}}\left|\operatorname{disc}\left([\mathbf{g}, \mathbf{h}), U, \mathcal{P}_{n, s}\right)\right|, \\
\sup _{\mathbf{g}, \mathbf{h} \in[0,1]^{s}} \gamma_{U}\left|\operatorname{disc}\left([\mathbf{g}, \mathbf{h}), U, \mathcal{P}_{n, s}\right)\right| \leq 2 \delta+\sup _{\mathbf{g}, \mathbf{h} \in \Gamma_{U}} \gamma_{U}\left|\operatorname{disc}\left([\mathbf{g}, \mathbf{h}), U, \mathcal{P}_{n, s}\right)\right| .
\end{gathered}
$$


For any positive integers $n$ and $s$, let $\mathcal{P}_{n, s}=\left\{\mathbf{x}^{1}, \ldots, \mathbf{x}^{n}\right\}$ be a set of independent and identically distributed random points uniformly distributed on $[0,1]^{s}$. The quantities $\operatorname{disc}\left([\mathbf{g}, \mathbf{h}), U,\left\{\mathbf{x}^{i}\right\}\right)$ are also iid random variables indexed by $\mathbf{g}, \mathbf{h}$, and $U$. Each of these random variables has zero mean and is bounded by \pm 1 . Moreover,

$$
\operatorname{disc}\left([\mathbf{g}, \mathbf{h}), U, \mathcal{P}_{n, s}\right)=\frac{1}{n} \sum_{i=1}^{n} \operatorname{disc}\left([\mathbf{g}, \mathbf{h}), U,\left\{\mathbf{x}^{i}\right\}\right)
$$

To get an upper bound on the local discrepancy function, Bennett's inequality (see, e.g., 8]) is used. Let $Y_{1}, \ldots, Y_{n}$ be independent random variables with zero means and bounded ranges: $\left|Y_{i}\right| \leq M$. Let $\sigma_{i}^{2}$ be variances of $Y_{i}$ and let $V$ be any number such that $V \geq \sum_{i=1}^{n} \sigma_{i}^{2}$. Then for each $\eta>0$, Bennett's inequality states that

$$
\operatorname{Prob}\left\{\left|Y_{1}+\cdots+Y_{n}\right| \geq \eta\right\} \leq 2 \exp \left(-\frac{\eta^{2}}{V} B(M \eta / V)\right)
$$

where $B(t)=t^{-2}((1+t) \ln (1+t)-t)$.

We shall use this inequality for $Y_{i}=\operatorname{disc}\left([\mathbf{g}, \mathbf{h}), U,\left\{\mathbf{x}^{i}\right\}\right)$, so we may take $M=1$ and $V=n$. Taking $\eta=n \delta_{U}$, it follows from above that $\eta^{2} / V=n \delta_{U}^{2}, M \eta / V=\delta_{U}$, and

$$
\operatorname{Prob}\left\{\left|\operatorname{disc}\left([\mathbf{g}, \mathbf{h}), U, \mathcal{P}_{n, s}\right)\right| \geq \delta_{U}\right\} \leq 2 \exp \left(-n \delta_{U}^{2} B\left(\delta_{U}\right)\right), \quad \forall \mathbf{g}, \mathbf{h} \in \Gamma_{U}
$$

Since it is assumed above that $\delta_{U}<1$, Lemma 1 implies that $B\left(\delta_{U}\right) \geq B(1)=$ $2 \ln (2)-1$. Since $\delta=(\hat{c} / n)^{b}$ for some $b<1 / 2$, we have $n=\hat{c} / \delta^{1 / b}$, and

$$
\operatorname{Prob}\left\{\left|\operatorname{disc}\left([\mathbf{g}, \mathbf{h}), U, \mathcal{P}_{n, s}\right)\right| \geq \delta_{U}\right\} \leq 2 \exp \left(-\left(\hat{c} B(1) / \gamma_{U}^{2}\right) \delta^{2-1 / b}\right), \quad \forall \mathbf{g}, \mathbf{h} \in \Gamma_{U}
$$

Note that $2-1 / b<0$.

For a given $U$, there are no more than

$$
\left(m_{U}-1\right)^{2|U|} \leq\left(|U| / \delta_{U}\right)^{2|U|}=\exp \left(2|U|\left[\ln (|U|)-\ln (\delta)+\ln \left(\gamma_{U}\right)\right]\right)
$$

possible values of $(\mathbf{g}, \mathbf{h}) \in \Gamma_{U} \times \Gamma_{U}$ with $\mathbf{g} \leq \mathbf{h}$. In view of Lemmas 2 and 3 one may then write

$$
\left(m_{U}-1\right)^{2|U|} \leq \exp \left(c_{1}-\ln (\delta)\left[c_{2}-c_{3} \ln (\delta)\right]\right) \leq \exp \left(\left(c_{4} B(1) / \hat{\gamma}^{2}\right) \delta^{2-1 / b}\right)
$$

for some positive constants $c_{1}, c_{2}, c_{3}$, and $c_{4}$ that are independent of $U, n$, and $\delta$, although $c_{4}$ depends on $b$.

Combining (13) and (14), it follows that

$\operatorname{Prob}\left\{\sup _{\mathbf{g}, \mathbf{h} \in \Gamma_{U}} \gamma_{U}\left|\operatorname{disc}\left([\mathbf{g}, \mathbf{h}), U, \mathcal{P}_{n, s}\right)\right| \geq \delta\right\} \leq 2 \exp \left(\left(-\hat{c}+c_{4}\right)\left(B(1) / \gamma_{U}^{2}\right) \delta^{2-1 / b}\right)$.

By choosing $\hat{c}>2 c_{4}$, one ensures that

$$
\operatorname{Prob}\left\{\sup _{\mathbf{g}, \mathbf{h} \in \Gamma_{U}} \gamma_{U}\left|\operatorname{disc}\left([\mathbf{g}, \mathbf{h}), U, \mathcal{P}_{n, s}\right)\right| \geq \delta\right\} \leq 2 \exp \left(-\left[\hat{c} B(1) /\left(2 \gamma_{U}^{2}\right)\right] \delta^{2-1 / b}\right) .
$$


To obtain a lower bound on the probability of having a low discrepancy set, one sums the above quantity over $U$ :

$$
\begin{aligned}
& \operatorname{Prob}\left\{\mathcal{D}_{\infty}\left(\mathcal{P}_{n, s}, \gamma\right) \geq 3 \delta\right\} \\
& \leq \operatorname{Prob}\left\{\sup _{U \text { with } \delta_{U} \leq 1} \sup _{\mathbf{g}, \mathbf{h} \in \Gamma_{U}} \gamma_{U}\left|\operatorname{disc}\left([\mathbf{g}, \mathbf{h}), U, \mathcal{P}_{n, s}\right)\right| \geq \delta\right\} \\
& \leq \sum_{U} 2 \exp \left(-\left[\hat{c} B(1) /\left(2 \gamma_{U}^{2}\right)\right] \delta^{2-1 / b}\right) \\
& \leq \sum_{U} \frac{2}{\exp \left(\left[\hat{c} B(1) /\left(2 \gamma_{U}^{2}\right)\right] \delta^{2-1 / b}\right)} \\
& \leq \sum_{U} \frac{2^{1+a / 2}(a /(2 e))^{a / 2} \gamma_{U}^{a} \delta^{a(1-2 b) /(2 b)}}{(\hat{c} B(1))^{a / 2}} \\
& \leq \frac{2^{1+a / 2}(a /(2 e))^{a / 2} \delta^{a(1-2 b) /(2 b)}}{(\hat{c} B(1))^{a / 2}} \sum_{U} \gamma_{U}^{a} \\
& \leq \frac{2^{1+a / 2}(a /(2 e))^{a / 2} \delta^{a(1-2 b) /(2 b)}}{(\hat{c} B(1))^{a / 2}} \prod_{j=1}^{\infty}\left(1+\gamma_{j}^{a}\right) .
\end{aligned}
$$

The product involving $\gamma_{j}^{a}$ is finite because of the summability condition on the $\gamma_{j}^{a}$. If $\delta>\hat{\gamma}$, then $\operatorname{Prob}\left\{\mathcal{D}_{\infty}\left(\mathcal{P}_{n, s}, \gamma\right) \geq 3 \delta\right\}=0$ automatically. Otherwise, $\delta^{a(1-2 b) /(2 b)} \leq$ $\hat{\gamma}^{a(1-2 b) /(2 b)}$ and the last term above may be made as small as desired by choosing $\hat{c}$ large enough. Doing so forces $\operatorname{Prob}\left\{\mathcal{D}_{\infty}\left(\mathcal{P}_{n, s}, \gamma\right)<3 \delta\right\}$ to be greater than zero, completing the proof.

\section{ACKNOWLEDGMENTs}

We would like to thank Youming Li and Erich Novak for valuable comments. The research has been partially supported by the Hong Kong Research Grants Council grant HKBU/2030/99P, the Hong Kong Baptist University Faculty Research Grant FRG/00-01/II-62, the Australian Research Council, and the National Science Foundation under Grant CCR-0095709.

\section{REFERENCES}

[1] M. Drmota and R.F. Tichy, Sequences, Discrepancies and Applications, Lecture Notes in Math. 1651, Springer, Berlin, 1997. MR 98j:11057

[2] S. Heinrich, E. Novak, G. W. Wasilkowski, and H. Woźniakowski, The inverse of the stardiscrepancy depends linearly on the dimension, Acta Arithmetica XCVI.3, pp. 279-302, 2001. MR 2002b: 11103

[3] F. J. Hickernell, I. H. Sloan, and G. W. Wasilkowski, On tractability of weighted integration over bounded and unbounded regions in $\mathbb{R}^{s}$, Math. Comp., posted on January 5, 2004, PII S 0025-5718(04)01624-2 (to appear in print).

[4] F. J. Hickernell, I. H. Sloan, and G. W. Wasilkowski, On tractability of integration for certain Banach spaces of functions, "Monte Carlo and Quasi-Monte Carlo Methods 2002" (H. Niederreiter, ed.), Springer, 2004, pp. 51-71.

[5] H. Niederreiter, Random Number Generation and quasi-Monte Carlo Methods, SIAM, Philadelphia, 1992. MR 93h:65008

[6] E. Novak, Deterministic and Stochastic Error Bounds in Numerical Analysis, Lecture Notes in Mathematics 1349, Springer, 1988. MR 90a:65004]

[7] E. Novak and H. Woźniakowski, Intractability results for integration and discrepancy, J. of Complexity 17, pp. 388-441, 2001. MR 2002f:65204 
[8] D. Pollard, Convergence of Stochastic Processes, Springer-Verlag, Berlin, 1984. MR 86i: 60074

[9] I. H. Sloan and H. Woźniakowski, When are quasi-Monte Carlo algorithms efficient for high dimensional integrals?, J. of Complexity 14, pp. 1-33, 1998. MR 99d:65384

[10] J.F. Traub, G. W. Wasilkowski, and H. Woźniakowski, Information-Based Complexity, Academic Press, New York, 1988. MR 90f:68085

Department of Mathematics, Hong Kong Baptist University, Kowloon Tong, HONG KONG

E-mail address: fred@math.hkbu.edu.hk

School of Mathematics, University of New South Wales, Sydney 2052, Australia

E-mail address: sloan@maths.unsw.edu.au

Department of Computer Science, University of Kentucky, 773 Anderson Hall, Lexington, Kentucky 40506-0046

E-mail address: greg@cs.uky.edu 